\title{
Anthracene Diphosphate Ligands for CdSe Quantum Dots; Molecular Design for Efficient Upconversion
}

Jonathan De Roo, ${ }^{\ddagger[a, b]}$ Zhiyuan Huang, ${ }^{f[c]}$ Nathaniel J. Schuster, ${ }^{[a]}$ Leslie S. Hamachi, ${ }^{[a]}$ Dan N. Congreve, ${ }^{[\mathrm{d}]}$ Zihao Xu, ${ }^{[\mathrm{e}]}$ Pan Xia ${ }^{[\mathrm{c}]}$, Dmitry A. Fishman, ${ }^{[\mathrm{f}]}$ Tianquan Lian, ${ }^{[\mathrm{e}]}$ Jonathan Owen, ${ }^{*[a]}$ Ming Lee Tang*[c]

[a] Department of Chemistry, Columbia University, 3000 Broadway, New York, NY 10027, USA

[b] Department of Chemistry, University of Basel, Mattenstrasse 24a BPR 1096, 4058 Basel, Switzerland

[c] Department of Chemistry, University of California Riverside, Riverside, CA 92521, USA

[d] Rowland Institute, Harvard University, Cambridge MA 02142, USA

[e] Department of Chemistry, Emory University, Atlanta, GA 30322, USA

[f] Department of Chemistry, University of California Irvine, Irvine, CA 92697, USA

$\neq \quad$ These authors contributed equally to this work

Corresponding authors:

Prof. Jonathan S. Owen: $\quad$ jso2115@ columbia.edu

Prof. Ming L. Tang: $\quad$ mltang@ucr.edu 


\begin{abstract}
Quantum dot (QD) sensitized photon upconversion follows a multi-step energy transfer process from QD to transmitter ligand to a soluble annihilator. Using a novel 10-R-anthracene-1,8diphosphoric acid ( $\mathrm{R}=$ octyl, 2-hexyldecyl, phenyl) ligand with high binding affinity for CdSe quantum dot (QD) surfaces, we demonstrate a photon upconversion process that is limited by the transmitter to annihilator transfer efficiency. Using ${ }^{1} \mathrm{H}$ NMR spectroscopy we demonstrate that these bidentate diphosphate ligands rapidly and irreversibly displace two carboxylate ligands. These ligands mediate energy transfer from the photoexcited QDs to a triplet annihilator (1,10-diphenylanthracene), producing overall photon upconversion quantum efficiencies as high as $17 \%$, the highest for QDs with no shells. Transient absorption spectroscopy shows that the ADP ligand supports a 3.4 fold longer triplet state lifetime compared to 9 -ACA $(299.9 \pm 9.5$ vs $88.2 \pm 2.1 \mu \mathrm{s})$, increasing the probability of the energy transfer.
\end{abstract}




\section{Main text}

To harness the high absorption cross section of semiconductor quantum dots (QDs) for energy harvesting, the photoexcited state should be directed away from the QD; perhaps by transmitters that would play a similar role to molecular antennas used in natural photosynthesis. Organic ligands bound to the QD are used to capture and transmit the photoexcited state. However, surface bound ligands and/or impurities can provide alternative pathways and reduce the energy transfer efficiency. ${ }^{1-2}$ Multi-step energy transfer schemes involving QDs must, therefore, balance the acceptor ligand binding strength, the QD solubility, as well as the driving force and surface electronic structure.

Photon upconversion processes, where two or more low energy photons are converted into a single high energy photon, have great potential in biological imaging and energy conversion. ${ }^{3-}$

${ }^{4}$ Recently, QDs (e.g., $\mathrm{CdSe}, \mathrm{CsPbBr}_{3}, \mathrm{PbS}$ ) have been used to photosensitize adsorbed aromatic transmitter ligands to effect efficient photon upconversion. ${ }^{5-8}$ The transmitter ligands (e.g., 9antracene carboxylic acid (9-ACA), Figure 1) must both accept the energy of QD excited state in the form of a long lived triplet state. ${ }^{5,9}$ and transfer that energy to a so called "annihilator" capable of bimolecular triplet fusion and fluorescence (e.g., diphenylanthracene (DPA), Figure 1). ${ }^{10,11}$ To date, photon upconversion quantum yields (PUQY) as high as $14 \%$ with CdSe, ${ }^{12}$ $12 \%$ with $\mathrm{PbS},{ }^{13}$ and $18.3 \%$ with core-shell $\mathrm{CuInS}_{2} / \mathrm{ZnS}$ QDs have been reported. ${ }^{14}$

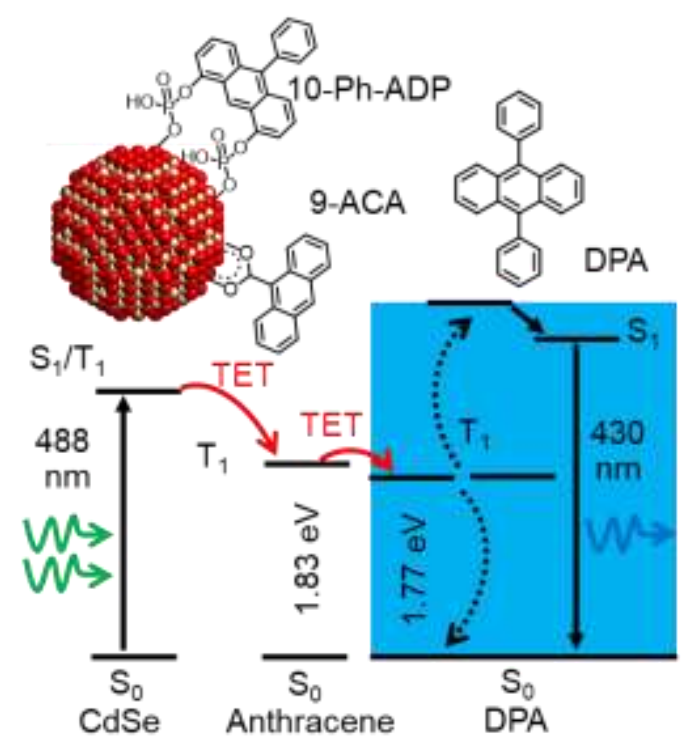

Figure 1. Illustration of energy transfer in this hybrid upconversion platform. Green and blue wavy arrows denote the photo excitation of CdSe QDs and the emission of upconverted photons from 9,10-diphenylanthracene (DPA), respectively. Red arrows show the triplet energy transfer (TET) of CdSe QDs to surface anchored anthracene (An) transmitter ligands 9-ACA or 10-Ph-ADP, and TET from transmitters to annihilators DPA. Black dotted arrows indicate the triplet-triplet annihilation of DPA. 
Although 9-ACA is the most successful transmitter ligand for upconversion with CdSe QDs, its interaction with the QD surface is poor. To improve the PUQY, we sought to enhance electronic coupling between the transmitter and the QD by designing bidentate transmitter ligands with high affinity for the QD surface. We reasoned that a multi-modal ligand scaffold based on a bidentate 1,8-anthracendiyl backbone would provide both high binding affinity for the surface and strong electronic coupling to the QD excited state. Multidentate ligands, including scaffolds made from citric acid, ${ }^{15}$ EDTA, ${ }^{16}$ or multidentate polymers, ${ }^{17}$ are known to have greater surface binding affinities than monodentate carboxylate ligands. However, carboxylate ligands, such as 9-ACA do not readily displace the native octadecylphosphonic acid (ODPA) from the surface of CdSe QDs. Therefore, a great excess of 9-ACA must be used to effect ligand exchange, and it is uncertain if the relatively weak binding influences the energy transfer efficiency. For this reason, our bidentate phosphate binding moiety enables precise and quantitative control over the ligand exchange reactivity, as opposed to a brute force displacement of the original surfactants via mass action. ${ }^{18-20}$

Here, we report three 10-R-anthracene-1,8-diphosphoric acid (ADP, R = octyl, 2hexyldecyl, phenyl) derivatives and show that their high affinity for CdSe QDs allows stoichiometric displacement of native ligands. The product results in highly efficient photon upconversion when using DPA as the annihilator. The improvement is attributed to a longer triplet life time on the ADP transmitter ligand and thus more efficient triplet energy transfer to the DPA emitter, showing that the transmitter structure is important to finely tune the elementary energy transfer steps in photon upconversion.

Our bidentate anthracene-based transmitter ligand incorporates two design features: (1) two phosphate groups with rotational flexibility for $\kappa_{2}$-surface coordination; and (2) a substituent in the 10-position that can enhance QD colloidal stability. These features can be incorporated by derivatizing 1,8-dihydroxyanthraquinone (1) as shown in Scheme 1. Nucleophilic addition of the desired $\mathrm{R}$ group at the 10-position is followed by an efficient sequence of reduction, dehydration, and aromatization of anthraquinone. ${ }^{21}$ tert-butyldimethylsilyl protecting groups prevent attack of the Grignard reagent at the 9-position ${ }^{22}$, providing 3 with near-perfect regioselectivity. Reduction of $\mathbf{3}$ with sodium borohydride and subsequent acidic work-up allows deprotection, dehydration, and rearomatization, providing the diol 4. Phosphorylation of the hydroxyl groups with the chlorophosphite ester, followed by dealkylation leads to ADP anthracene. 


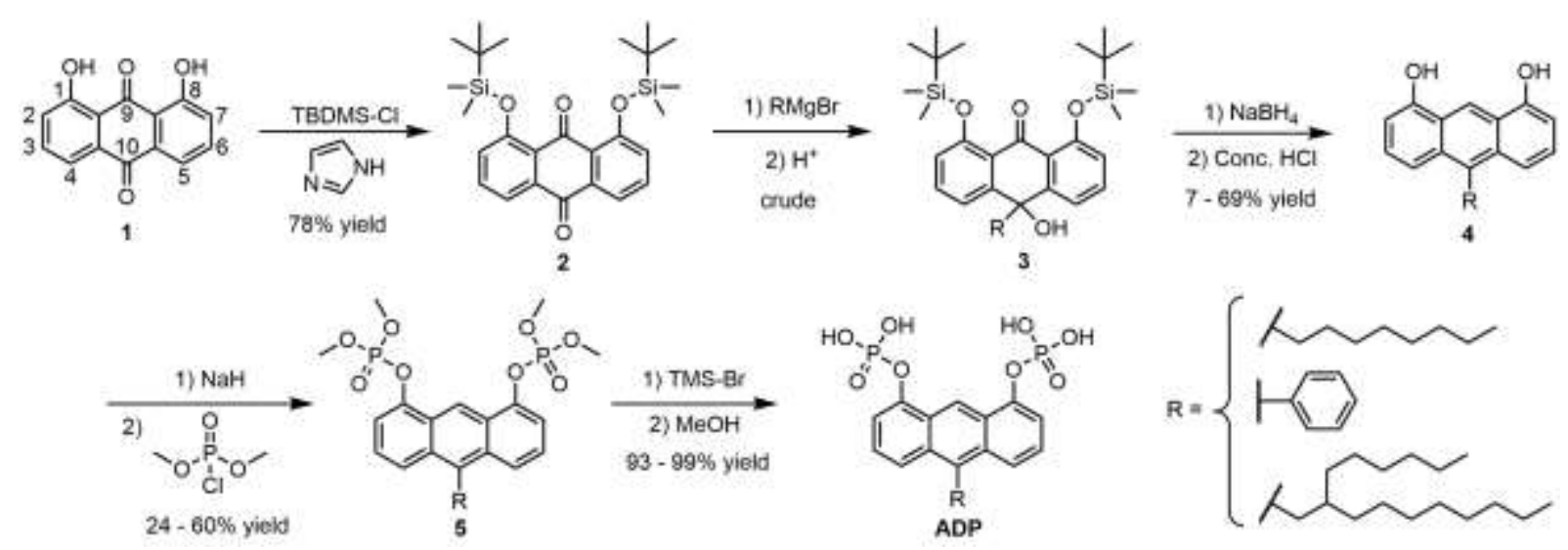

Scheme 1. Synthesis of anthracene dihydrogen phosphate (ADP) derivatives.

The 10-n-octyl, phenyl and the (2-hexyl)-n-decyl ADP derivatives have similar optical absorption and photoluminescence spectra (Figure S1) but different photoluminescence quantum yields ((PLQY), $38 \%, 68 \%$ and $76 \%$ for the $n$-octyl, 2-hexyldecyl and phenyl derivatives respectively). The higher PLQY of the 10-Ph-ADP derivative is likely due to the rigid benzene ring and a lower non-radiative decay rate for both the emissive singlet and dark triplet state, a property that may enhance the PUQY. ${ }^{5}$

ADP readily displaces carboxylic acids from CdSe QDs as shown in Figure 2A. ${ }^{23}$ The broadened ${ }^{1} \mathrm{H}$ NMR resonances of bound oleate ligands can be distinguished from the sharp signals of free ligands following the reaction with ADP.${ }^{24}$ Upon addition of 10-(2-hexyl)decylADP (0.6 equivalents with respect to bound oleate), the oleate resonances sharpen, indicating the displacement of oleate from the QD surface. After purification, the oleate resonances are absent from the ${ }^{1} \mathrm{H}$ NMR spectrum, while the broadened $\mathrm{CH}_{2}$ and $\mathrm{CH}_{3}$ resonances of the 10-(2hexyl)decyl-ADP remain (Figure 2C). The aromatic signals are strongly broadened due to their proximity to the surface and are hardly detected. ${ }^{25}$ The two methyl groups of the (2-hexyl)decyl chain are inequivalent and have different line widths that is typical for branched ligands and is due to different rotational degrees of freedom and solvation. ${ }^{26-27}$ The ${ }^{31} \mathrm{P}$ NMR spectrum of the CdSe QDs bound by 10-(2-hexyl)decyl-ADP (Figure 2C, inset) features a broad resonance ( $\delta$ $=-0.5 \mathrm{ppm})$ whose FWHM $(1714 \mathrm{~Hz})$ is comparable to that typical of phosphonate ligands bound to $\mathrm{CdSe}^{27}$ Given the stoichiometry of the exchange (0.6 eq ADP) and the complete removal of oleate, we infer that the exchange is quantitative and that both phosphate groups bind the QD surface. Compounds 10- $\boldsymbol{n}$-octyl-ADP and 10-phenyl-ADP have similar reactivity but do not support a colloidal dispersion upon complete exchange and purification. To assess the binding affinity of the ADP framework, thiol ligands were added and ADP displacement was studied with ${ }^{31} \mathrm{P}$ NMR. ${ }^{28}$ After adding 75 equivalents of $n$-hexanethiol to a solution of ADP 
bound QDs, the 10-(2-hexyl)decyl-ADP ${ }^{31}$ P NMR signal remains broad, evidence that the ADP ligand remains bound (Figure S2). However, complete exchange occurs upon adding triethylamine to this mixture (Figure S2). Unlike the binding of 9-ACA, where exchange is generally not complete, and the QD PL is mostly quenched, binding of ADP completely quenches the QD PL, even at lower ligand loadings used in the upconversion measurements described below. This increased quenching may be partially caused by other charge trap states introduced by the phosphate binding (see below). ${ }^{20}$ Each of these observations supports the efficient displacement of the starting carboxylic acid ligands and the tight binding of the ADP framework to the QD surface.
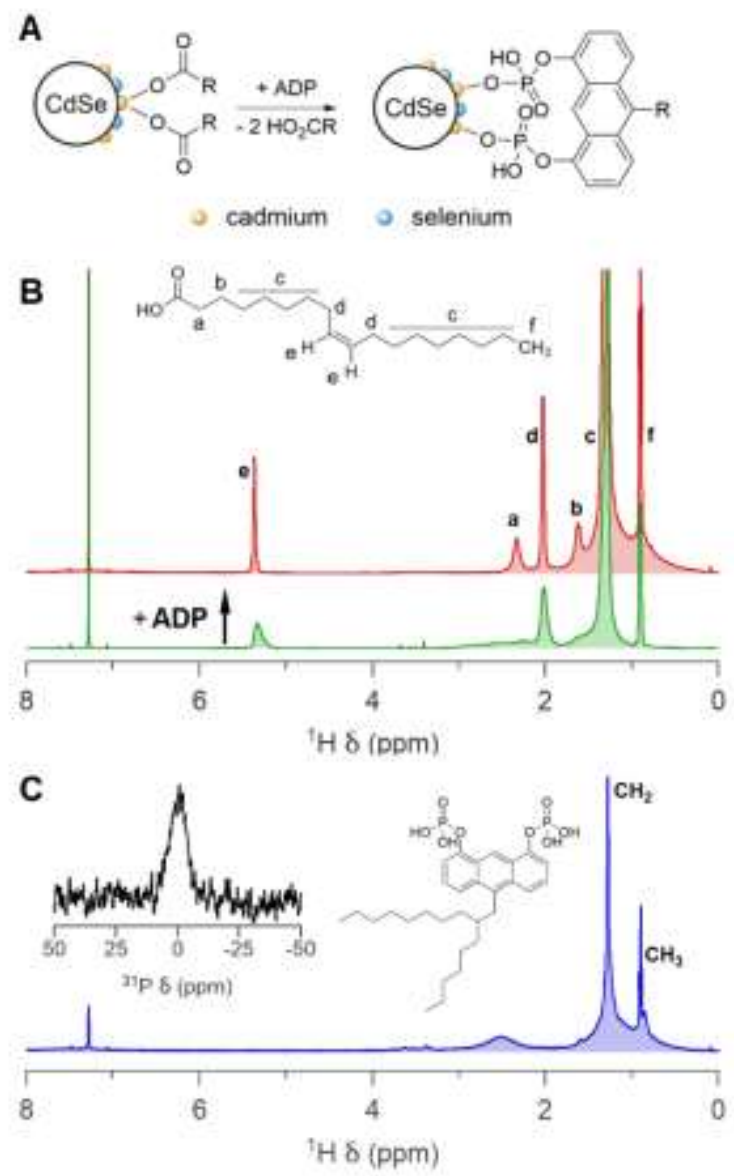

Figure 2. (a) Scheme of ADP-for-oleate exchange on CdSe QDs. (b) ${ }^{1} \mathrm{H}$ NMR spectrum of oleate capped CdSe QDs in $\mathrm{CDCl}_{3}$, before and after addition of 0.6 eq of 10-(2-hexyl)decyl-ADP, [QD] $=150 \mathrm{mM}$. (c) ${ }^{1} \mathrm{H}$ NMR spectrum after purification. The inset shows the ${ }^{31} \mathrm{P}$ NMR spectrum.

A three-component upconversion platform was assembled from a wurtzite $\mathrm{CdSe}^{29-30} \mathrm{QD}$ absorber (diameter $=2.4 \mathrm{~nm}$, native ligands $=$ ODPA), with a coverage of surface bound 10Ph-ADP or 9-ACA transmitter ligands that optimizes the upconversion yield and maintains colloidal stability, and a DPA emitter. Photoexcitation $(\lambda=488 \mathrm{~nm})$ and energy transfer to the 
surface anchored 10-Ph-ADP or 9-ACA ligands leads to ligand centered triplets that transfer their energy to the DPA (Figure 1). Two DPA triplets undergo annihilation, producing $430 \mathrm{~nm}$ fluorescence. Figure 3 shows the absorption and emission spectra of 9-ACA, 10-Ph-ADP, DPA and wurtzite CdSe QDs. Selective photoexcitation of the QDs at $488 \mathrm{~nm}$ leads to upconversion and fluorescence from DPA in the 400-450 nm spectral range. Partial displacement of the ODPA with 10-Ph-ADP leads to CdSe QDs whose PUQY increases up to a maximum value and then decreases (see Figure S3).

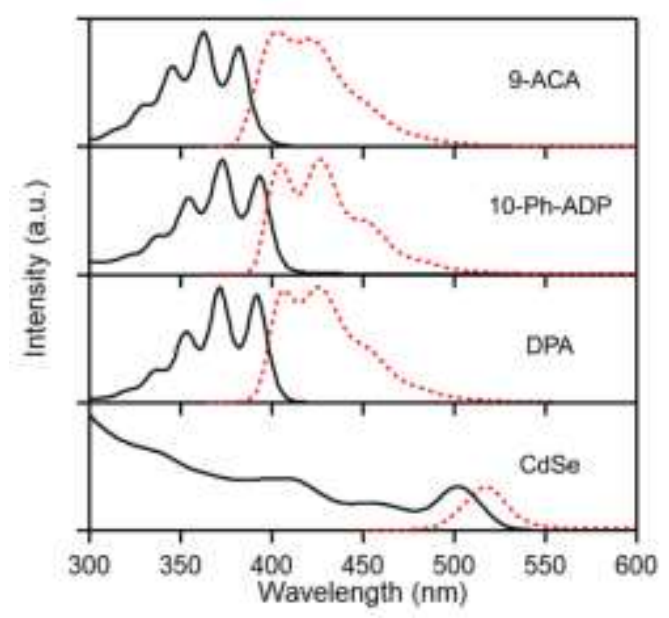

Figure 3. Absorption and emission spectra of 9-ACA, 10-Ph-ADP, DPA and 2.4 nm diameter wurtzite CdSe QDs. All spectra were obtained in hexanes at room temperature.

At optimal ligand loadings (5-8 anthracene ligands per particle), CdSe QDs with 10Ph-ADP ligands achieve a PUQY of 16.9\%, 1.33 times higher than possible with 9-ACA (Figure S3). As presented in Figure 3 and Table 1, the absorption onset of 10-Ph-ADP is redshifted by $11 \mathrm{~nm}(0.091 \mathrm{eV})$ compared with 9-ACA. A similar shift in the $\mathrm{T}_{1}$ energy for 10-PhADP would provide a larger driving force for energy transfer from the CdSe QDs, lowering the probability of back transfer and therefore increasing the PUQY. Additionally, the parent acid of 10-Ph-ADP has a fluorescence quantum yield of 76\%, 1.8 times higher than that for 9-ACA, indicating diminished non-radiative decay in the former. The rigidity of 10-Ph-ADP reduces vibrational losses and may enhance the PUQY observed when compared to the 9-ACA transmitter ligand. 
Table 1. Key parameters of ligands 9-ACA, 10-Ph-ADP, and QDs used in upconversion experiments. Fluorescence quantum yield, $\Phi_{\mathrm{PL}}$, lowest energy absorption peak, $\lambda_{\mathrm{abs}}$, number of bound ligands per CdSe QD, n, upconversion quantum yield, $\Phi_{\mathrm{UC}}$, weighted average triplet energy transfer rate, $\mathrm{k}_{\mathrm{TET}}$, and efficiency, $\Phi_{\mathrm{TET}}$.

\begin{tabular}{lcccccc}
\hline & $\begin{array}{c}\Phi_{\mathrm{PL}} \\
(\%)\end{array}$ & $\begin{array}{c}\lambda_{\mathrm{abs}} \\
(\mathrm{nm})\end{array}$ & $n$ & $\begin{array}{c}\Phi_{\mathrm{UC}} \\
(\%)\end{array}$ & $\begin{array}{c}\mathrm{k}_{\mathrm{TET}} \\
\left(\mathrm{s}^{-1}\right)\end{array}$ & $\begin{array}{c}\Phi_{\mathrm{TET}} \\
(\%)\end{array}$ \\
\hline 9-ACA & 43.2 & 382 & & & & \\
10-Ph-ADP & 76.0 & 393 & & & & \\
CdSe/9-ACA & 2.1 & 382 & 7.4 & 12.7 & $(5.98 \pm 0.18) \times 10^{10}$ & $84.7 \pm 5.0$ \\
CdSe/10-Ph-ADP & $<0.1$ & 393 & 5.8 & 16.9 & $(1.22 \pm 0.02) \times 10^{11}$ & $86.4 \pm 5.1$ \\
\hline
\end{tabular}

Transient absorption (TA) spectroscopy was used to study the TET from CdSe QDs to surface anchored 9-ACA and 10-Ph-ADP (Figure 4). The $\mathrm{T}_{1}-\mathrm{T}_{\mathrm{n}}$ transition of 9-ACA is reported to occur at $433 \mathrm{~nm}$, which overlaps with the excited state absorption of CdSe QDs. The absorption from photoexcited CdSe/ODPA was subtracted from the TA spectra after normalizing to the signal at $457 \mathrm{~nm}$ (where there is no contribution from 9-ACA or 10-Ph-ADP $\mathrm{T}_{1}$ triplet state). The resulting double difference spectra of the CdSe/9-ACA (Figure 5a) show an absorption band centered at $\sim 430 \mathrm{~nm}$, corresponding to the $\mathrm{T}_{1}-\mathrm{T}_{\mathrm{n}}$ absorption of 9-ACA $\mathrm{T}_{1}$ triplet state. ${ }^{10,31}$ Similarly, the double difference spectra of CdSe/10-Ph-ADP (Figure 5b) shows an absorption peak at $\sim 435 \mathrm{~nm}$, which can be attributed to the $\mathrm{T}_{1}-\mathrm{T}_{\mathrm{n}}$ absorption of 10Ph-ADP $\mathrm{T}_{1}$ triplet state. These triplet populations grow on the 1 ps to 3 ns time frame, concomitant with the decay of QD exciton bleach, consistent with the formation of the ligand centered triplet state by triplet energy transfer from the QD.
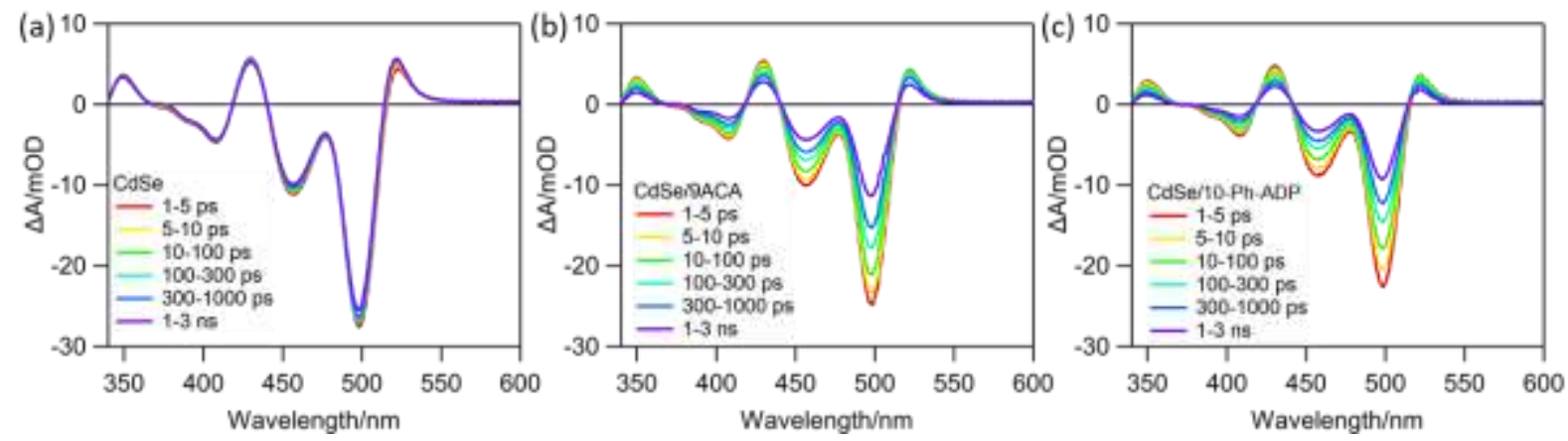

Figure 4. Femtosecond transient absorption spectra of (a) CdSe/ODPA, and (b) CdSe with surface anchored 9ACA and (c) CdSe with 10-Ph-ADP. Samples are dissolved in hexanes and excited with a $505 \mathrm{~nm}$ pulsed laser at RT. 

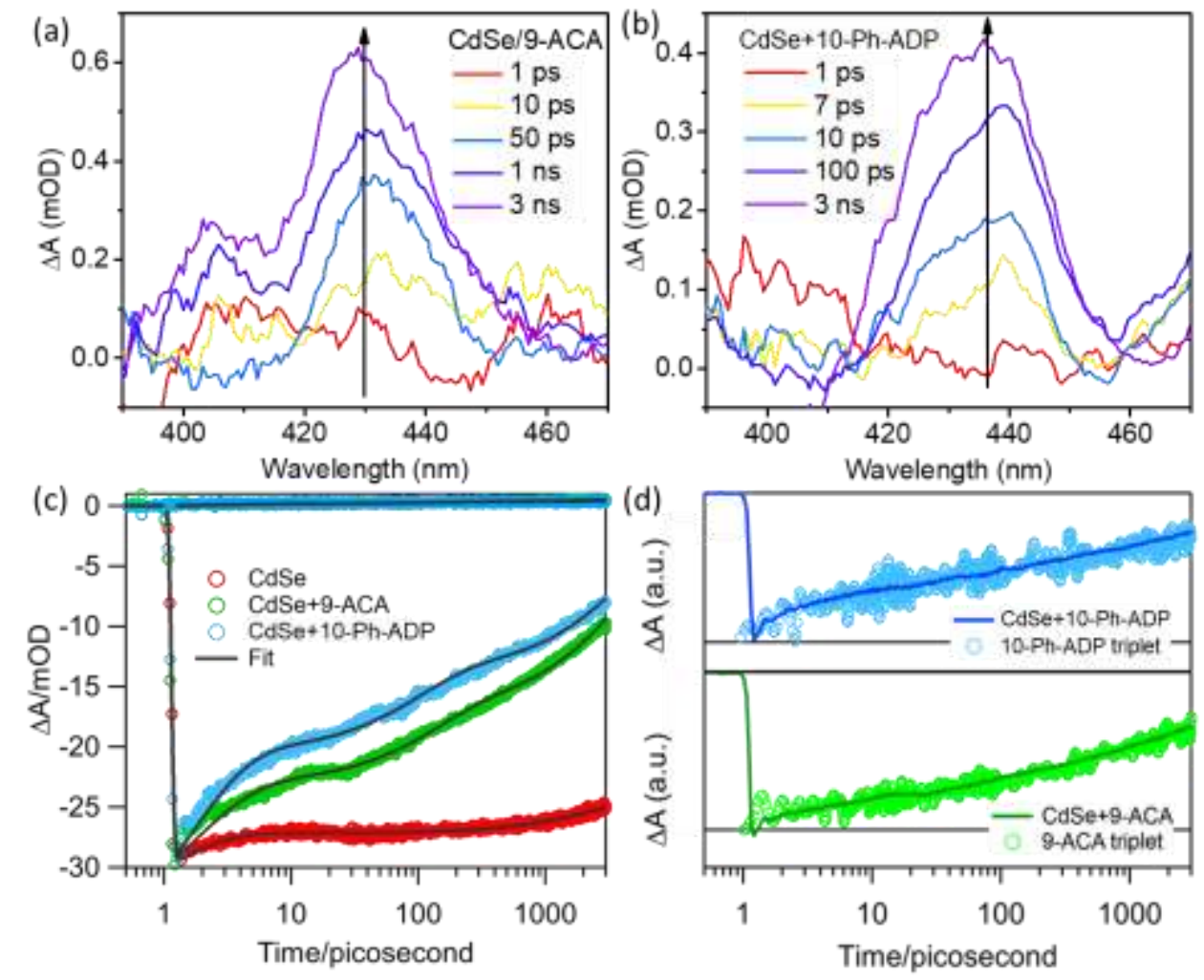

Figure 5. Double difference spectra constructed by subtracting the CdSe/ODPA TA spectrum from the transient spectra of (a) CdSe/9-ACA and (b) CdSe/10-Ph-ADP. (c) The kinetics of QD exciton bleach at $\sim 500 \mathrm{~nm}$ are plotted and fit with the model described in the supporting information. (d) Comparison of the kinetics at $\sim 500 \mathrm{~nm}$ (scaled) and triplet signal at $\sim 430 \mathrm{~nm}$ of 9-ACA and 10-Ph-ADP. This shows the triplet formation follows the CdSe exciton decay. The triplet signal before $1 \mathrm{ps}$ is discarded due to the noise.

Detailed analysis of the kinetics of triplet formation and QD excited state decay was carried out to obtain the rates and efficiencies of triplet energy transfer from CdSe QDs to 10-Ph-ADP and 9-ACA. The recovery of the CdSe excitonic bleach for CdSe/ODPA, CdSe/9-ACA and CdSe/10-Ph-ADP are compared in Figure 5c. The bleach recovery is fastest in the presence of 10-Ph-ADP and 9-ACA, which can be attributed to energy from the CdSe QD to 10-Ph-ADP and 9-ACA ligands. As shown in Figure 5d, the kinetics of 10-Ph-ADP and 9-ACA triplet formation follows the disappearance of the exciton in the CdSe QD. Therefore, the triplet energy transfer rate is equal to and can be extracted from the CdSe exciton bleach recovery kinetics. Details of the kinetic model and fitting parameters are presented in the Supporting Information. The fitting results show that the TET rate constant from CdSe to 10-Ph-ADP is $1.22 \times 10^{11} \mathrm{~s}^{-1}$, twice the value of $5.98 \times 10^{10} \mathrm{~s}^{-1}$ for 9-ACA (Table 1). The increased rate of energy transfer can be explained by the slightly lower energy of the $\mathrm{T}_{1}$ state of 10-Ph-ADP discussed above. Despite the faster TET rate constant, 10-Ph-ADP and 9-ACA show nearly the same 
TET efficiency in this $3 \mathrm{~ns}$ window, $\Phi_{\mathrm{TET}} \sim 85 \%$ (Table 1), presumably because both rates are significantly faster than recombination of the CdSe exciton (red curve in Figure 5c).

The threshold intensity for the cross-over from the quadratic to the linear regime is $492 \mathrm{~mW} / \mathrm{cm}^{2}$ for 9-ACA compared to $163 \mathrm{~mW} / \mathrm{cm}^{2}$ for 10-Ph-ADP when the optical densities for both samples at the excitation are 0.303 and 0.242 respectively (Fig. S4). Therefore, assuming the same rate and efficiency of DPA triplet-triplet annihilation, we infer that the triplet energy transfer from surface bound 10-Ph-ADP to free DPA in solution is at least three times more efficient compared to $\mathbf{9 - A} \mathbf{C A}^{32}$. This is consistent with a much longer lifetime of the $\mathrm{T}_{1}$ state of the 10-Ph-ADP transmitter $(299.9 \pm 9.5 \mu \mathrm{s})$ compared to that for 9-ACA $(88.2 \pm 2.1 \mu \mathrm{s})$. Overall this supports a higher overall upconversion efficiency, ${ }^{33-35}$ as the overall triplet energy transfer efficiency is increased by both the rate of TET and the transmitter triplet lifetime. The threshold intensity value is the point where the photon flux just replenishes the loss of the triplet excited states (due to various non-radiative loss processes + TTA processes which lead to upconversion). A longer transmitter lifetime thus translates into a lower threshold intensity for the transition from the quadratic to the linear regime. The reasons for this lifetime increase are unclear, but could be related to the lower rates of nonradiative recombination in the parent ADP framework, or rigidity that is enhanced by chelation.

In conclusion, we demonstrated that bidentate anthracene diphosphate ligands strongly bind CdSe QDs. Compared to monodentate ligands that coordinate weakly, this high binding affinity allows the ligand loading to be finely controlled. Similar to 9-carboxylic acid anthracene, these tightly bound transmitter ligands display CdSe to ligand triplet transfer efficiencies close to unity. A 33\% improvement in the overall PUQY is proposed to arise from a $\mathrm{T}_{1}$ lifetime that is 3.4 fold longer than 9-ACA when anchored on the CdSe surface. This study shows that transmitter ligand structure is critical to boosting the photon upconversion quantum yields and lowering the threshold intensities needed to access the linear regime. Future work should focus on identifying and eliminating deleterious triplet quenching sites on QDs and using molecular engineering to design ligands with long-lived triplet excited states that can efficiently accept photogenerated excitons.

\section{Experimental Section}

See Supporting information. 


\section{Acknowledgements}

The authors acknowledge Columbia University, Fulbright, the Belgian American Education Foundation, the Rowland Fellowship at the Rowland Institute at Harvard, the US National Science Foundation (CHE-1710352, 1532125), Horizon 2020 (COMPASS, No. 691185), and the US Department of Energy (DE-SC0018969 and DE-FG02-12ER16347) for financial support. 


\section{References}

1. Giansante, C.; Infante, I., Surface Traps in Colloidal Quantum Dots: A Combined Experimental and Theoretical Perspective. The Journal of Physical Chemistry Letters 2017, 5209-5215.

2. Kirkwood, N.; Monchen, J. O. V.; Crisp, R. W.; Grimaldi, G.; Bergstein, H. A.; du Fossé, I.; van der Stam, W.; Infante, I.; Houtepen, A. J., Finding and fixing traps in II-VI and III-V colloidal quantum dots: The importance of Z-type ligand passivation. J. Am. Chem. Soc. 2018.

3. Smith, B. R.; Gambhir, S. S., Nanomaterials for In Vivo Imaging. Chem Rev 2017, 117 (3), 901-986.

4. Ravetz, B. D.; Pun, A. B.; Churchill, E. M.; Congreve, D. N.; Rovis, T.; Campos, L. M., Photoredox catalysis using infrared light via triplet fusion upconversion. Nature 2019, 565 (7739), 343-346.

5. Huang, Z.; Tang, M. L., Designing Transmitter Ligands That Mediate Energy Transfer between Semiconductor Nanocrystals and Molecules. J. Am. Chem. Soc. 2017, 139 (28), 9412-9418.

6. He, S.; Luo, X.; Liu, X.; Li, Y.; Wu, K., Visible-to-Ultraviolet Upconversion Efficiency above 10\% Sensitized by Quantum-Confined Perovskite Nanocrystals. The Journal of Physical Chemistry Letters 2019, 5036-5040.

7. Wieghold, S.; Bieber, A. S.; VanOrman, Z. A.; Daley, L.; Leger, M.; Correa-Baena, J.-P.; Nienhaus, L., Triplet Sensitization by Lead Halide Perovskite Thin Films for Efficient Solid-State Photon Upconversion at Subsolar Fluxes. Matter 2019, 1 (3), 705-719.

8. Mase, K.; Okumura, K.; Yanai, N.; Kimizuka, N., Triplet sensitization by perovskite nanocrystals for photon upconversion. Chemical Communications 2017, 53 (59), 8261-8264. 9. Mongin, C.; Moroz, P.; Zamkov, M.; Castellano, F. N., Thermally activated delayed photoluminescence from pyrenyl-functionalized CdSe quantum dots. Nat. Chem. 2018, 10 (2), 225-230.

10. Mongin, C.; Garakyaraghi, S.; Razgoniaeva, N.; Zamkov, M.; Castellano, F. N., Direct observation of triplet energy transfer from semiconductor nanocrystals. Science 2016, 351 (6271), 369-372.

11. Huang, Z.; Li, X.; Mahboub, M.; Hanson, K. M.; Nichols, V. M.; Le, H.; Tang, M. L.; Bardeen, C. J., Hybrid Molecule-Nanocrystal Photon Upconversion Across the Visible and Near-Infrared. Nano Lett. 2015, 15 (8), 5552-5557.

12. Li, X.; Fast, A.; Huang, Z. Y.; Fishman, D. A.; Tang, M. L., Complementary Lockand-Key Ligand Binding of a Triplet Transmitter to a Nanocrystal Photosensitizer. Angew. Chem.-Int. Edit. 2017, 56 (20), 5598-5602.

13. Huang, Z.; Simpson, D. E.; Mahboub, M.; Li, X.; Tang, M. L., Ligand enhanced upconversion of near-infrared photons with nanocrystal light absorbers. Chemical Science 2016, 7 (7), 4101-4104.

14. Han, Y.; He, S.; Luo, X.; Li, Y.; Chen, Z.; Kang, W.; Wang, X.; Wu, K., Triplet Sensitization by "Self-Trapped" Excitons of Nontoxic CuInS2 Nanocrystals for Efficient Photon Upconversion. J. Am. Chem. Soc. 2019, 141 (33), 13033-13037.

15. Bishop, L. M.; Yeager, J. C.; Chen, X.; Wheeler, J. N.; Torelli, M. D.; Benson, M. C.; Burke, S. D.; Pedersen, J. A.; Hamers, R. J., A Citric Acid-Derived Ligand for Modular Functionalization of Metal Oxide Surfaces via "Click" Chemistry. Langmuir 2012, 28 (2), 1322-1329.

16. Hellstern, M.; Gantenbein, M.; Le Pleux, L.; Puebla-Hellmann, G.; Lörtscher, E.; Mayor, M., Electrochemical Multiplexing: Control over Surface Functionalization by Combining a Redox-Sensitive Alkyne Protection Group with "Click"-Chemistry. Advanced Materials Interfaces 2019, 6 (5). 
17. Du, L.; Wang, W. T.; Zhang, C. Q.; Jin, Z. C.; Palui, G.; Mattoussi, H., A Versatile Coordinating Ligand for Coating Semiconductor, Metal, and Metal Oxide Nanocrystals.

Chem. Mat. 2018, 30 (20), 7269-7279.

18. Huang, Z.; Li, X.; Yip, B. D.; Rubalcava, J. M.; Bardeen, C. J.; Tang, M. L.,

Nanocrystal Size and Quantum Yield in the Upconversion of Green to Violet Light with CdSe and Anthracene Derivatives. Chem. Mat. 2015, 27 (21), 7503-7507.

19. Kroupa, D. M.; Arias, D. H.; Blackburn, J. L.; Carroll, G. M.; Granger, D. B.; Anthony, J. E.; Beard, M. C.; Johnson, J. C., Control of Energy Flow Dynamics between Tetracene Ligands and PbS Quantum Dots by Size Tuning and Ligand Coverage. Nano Lett 2018, 18 (2), 865-873.

20. Knauf, R. R.; Lennox, J. C.; Dempsey, J. L., Quantifying Ligand Exchange Reactions at CdSe Nanocrystal Surfaces. Chem Mater 2016, 28 (13), 4762-4770.

21. Méry, S.; Haristoy, D.; Nicoud, J.-F.; Guillon, D.; Monobe, H.; Shimizu, Y., Liquid crystals containing a 2,6-disubstituted anthracene core-mesomorphism, charge transport and photochemical properties. J. Mater. Chem. 2003, 13 (7), 1622-1630.

22. Bakola-christianopoulou, M. N.; Apazidou, K. K., SYNTHESIS OF

HYDROLYTICALLY STABLE tert-BUTYLDIMETHYLSILYL ETHERS OF

HYDROXYANTHRAQUINONES. Phosphorus, Sulfur, and Silicon and the Related Elements 1996, 113 (1-4), 245-253.

23. Chen, O.; Chen, X.; Yang, Y.; Lynch, J.; Wu, H.; Zhuang, J.; Cao, Y. C., Synthesis of Metal-Selenide Nanocrystals Using Selenium Dioxide as the Selenium Precursor. Angew.

Chem., Int. Ed. 2008, 47 (45), 8638-8641.

24. Hens, Z.; Martins, J. C., A Solution NMR Toolbox for Characterizing the Surface Chemistry of Colloidal Nanocrystals. Chem Mater 2013, 25 (8), 1211-1221.

25. De Roo, J.; Coucke, S.; Rijckaert, H.; De Keukeleere, K.; Sinnaeve, D.; Hens, Z.; Martins, J. C.; Van Driessche, I., Amino Acid-Based Stabilization of Oxide Nanocrystals in Polar Media: From Insight in Ligand Exchange to Solution 1H NMR Probing of ShortChained Adsorbates. Langmuir 2016, 32 (8), 1962-1970.

26. De Roo, J.; Yazdani, N.; Drijvers, E.; Lauria, A.; Maes, J.; Owen, J. S.; Van Driessche, I.; Niederberger, M.; Wood, V.; Martins, J. C.; Infante, I.; Hens, Z., Probing Solvent-Ligand Interactions in Colloidal Nanocrystals by the NMR Line Broadening. Chem Mater 2018, 30 (15), 5485-5492.

27. De Roo, J.; Zhou, Z.; Wang, J.; Deblock, L.; Crosby, A. J.; Owen, J. S.; Nonnenmann, S. S., Synthesis of Phosphonic Acid Ligands for Nanocrystal Surface Functionalization and Solution Processed Memristors. Chem. Mat. 2018, 30 (21), 8034-8039.

28. Owen, J. S.; Park, J.; Trudeau, P. E.; Alivisatos, A. P., Reaction chemistry and ligand exchange at cadmium-selenide nanocrystal surfaces. J. Am. Chem. Soc. 2008, 130 (37), 12279-12280.

29. Carbone, L.; Nobile, C.; De Giorgi, M.; Sala, F. D.; Morello, G.; Pompa, P.; Hytch, M.; Snoeck, E.; Fiore, A.; Franchini, I. R., Synthesis and micrometer-scale assembly of colloidal $\mathrm{CdSe} / \mathrm{CdS}$ nanorods prepared by a seeded growth approach. Nano letters $\mathbf{2 0 0 7 , 7}$ (10), 2942-2950.

30. Yu, W. W.; Qu, L.; Guo, W.; Peng, X., Experimental determination of the extinction coefficient of CdTe, CdSe, and CdS nanocrystals. Chemistry of Materials 2003, 15 (14), 2854-2860.

31. Hirayama, S., Effect of substituent on the behaviour of the excited singlet and triplet states in carbonyl derivatives of anthracene of the type 9-X.CO·A. Journal of the Chemical Society, Faraday Transactions 1: Physical Chemistry in Condensed Phases 1982, 78 (8), 2411-2421. 
32. Monguzzi, A.; Tubino, R.; Hoseinkhani, S.; Campione, M.; Meinardi, F., Low power, non-coherent sensitized photon up-conversion: modelling and perspectives. Phys. Chem. Chem. Phys. 2012, 14 (13), 4322-4332.

33. Garakyaraghi, S.; Mongin, C.; Granger, D. B.; Anthony, J. E.; Castellano, F. N., Delayed Molecular Triplet Generation from Energized Lead Sulfide Quantum Dots. The Journal of Physical Chemistry Letters 2017, 8 (7), 1458-1463.

34. Huang, Z.; Xu, Z.; Mahboub, M.; Li, X.; Taylor, J. W.; Harman, W. H.; Lian, T.;

Tang, M. L., PbS/CdS Core-Shell Quantum Dots Suppress Charge Transfer and Enhance Triplet Transfer. Angewandte Chemie International Edition 2017, 56 (52), 16583-16587.

35. Huang, Z.; Xu, Z.; Mahboub, M.; Liang, Z.; Jaimes, P.; Xia, P.; Graham, K. R.; Tang, M. L.; Lian, T., Enhanced Near-Infrared-to-Visible Upconversion by Synthetic Control of PbS Nanocrystal Triplet Photosensitizers. J. Am. Chem. Soc. 2019, 141 (25), 9769-9772. 
Table of Contents

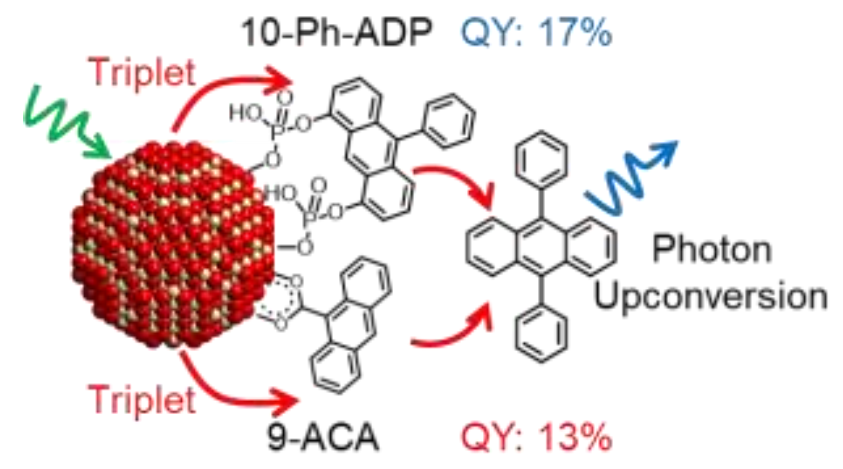

